Swarthmore College

Works

3-26-2001

\title{
Turbulent Conductivity Measurements In A Spherical Liquid Sodium Flow
}

Amy Reighard , '01

Michael R. Brown

Swarthmore College, doc@swarthmore.edu

Follow this and additional works at: https://works.swarthmore.edu/fac-physics

Part of the Physics Commons

Let us know how access to these works benefits you

\section{Recommended Citation}

Amy Reighard, '01 and Michael R. Brown. (2001). "Turbulent Conductivity Measurements In A Spherical Liquid Sodium Flow". Physical Review Letters. Volume 86, Issue 13. 2794-2797. DOI: 10.1103/

PhysRevLett.86.2794

https://works.swarthmore.edu/fac-physics/94

This work is brought to you for free by Swarthmore College Libraries' Works. It has been accepted for inclusion in Physics \& Astronomy Faculty Works by an authorized administrator of Works. For more information, please contact myworks@swarthmore.edu. 


\title{
Turbulent Conductivity Measurements in a Spherical Liquid Sodium Flow
}

\author{
A. B. Reighard and M. R. Brown \\ Department of Physics and Astronomy, Swarthmore College, Swarthmore, Pennsylvania 19081-1397
}

(Received 10 August 2000)

\begin{abstract}
We report the first measurement of a reduction in the conductivity of liquid sodium due to turbulence in a spherical flow (the $\beta$ effect). The sodium is contained in a $0.15 \mathrm{~m}$ diameter sphere, typical flow speeds are about $1 \mathrm{~m} / \mathrm{s}$, and magnetic Reynold's numbers range from 1 to 8 . We find a reduction from the molecular value of the conductivity of about $4 \%$. Results are in rough agreement with simple predictions from mean-field electrodynamics.
\end{abstract}

DOI: $10.1103 /$ PhysRevLett.86.2794

A magnetofluid dynamo is the process by which turbulent fluid kinetic energy is converted to magnetic energy through the $\mathbf{v} \times \mathbf{B}$ term in Ohm's law. A key element to the dynamo problem in astrophysical contexts is that many scenarios require a reduction in the conductivity or an enhancement of the diffusivity due to turbulence (the $\beta$ effect) to release the generated flux [1]. Understanding the turbulent $\beta$ effect is of paramount importance in several models. First, enhanced turbulent diffusivity is needed to explain the net azimuthal flux leaving the Sun [2,3]. In order for a flux rope to escape, virtually all the magnetofluid material must be decoupled from the magnetic fields by turbulent diffusivity. Second, competition between high conductivity and turbulent diffusivity at the base of the convection zone is critical for the theory of "interface dynamos" [4].

There has been significant progress in recent laboratory liquid sodium experiments designed to study the growth and decay of dynamo magnetic fields [5-9]. One can write the induction equation

$$
\partial \mathbf{B} / \partial t=\nabla \times(\mathbf{v} \times \mathbf{B})+\left(\eta_{0} / \mu_{0}\right) \nabla^{2} \mathbf{B}
$$

as an eigenvalue value equation

$$
\lambda \mathbf{B}=\Lambda \mathbf{B},
$$

where the operator $\Lambda=\nabla \times \mathbf{v} \times+\left(\eta_{0} / \mu_{0}\right) \nabla^{2}$, the eigenvalue $\lambda>0$ represents a growing eigenmode, and $\lambda<0$ represents a decaying eigenmode. Here $\eta_{0}$ is the molecular resistivity $\left(\eta_{0}=1 / \sigma_{0}\right)$. The eigenvalue equation simply reflects a competition between the convective and diffusive terms of Eq. (1). The Riga group recently reported a growing magnetic eigenmode in a long $(L=3 \mathrm{~m})$, thin $(r=0.2 \mathrm{~m})$ vortical sodium flow above a critical flow speed [7]. They initialized their experiment with a prescribed seed magnetic field and required a very specific helical flow field. Above a critical Reynold's number, they observed a slowly growing eigenmode mixed with an amplified external signal, while below the critical value, they observed decay of the eigenmode. The Maryland group recently reported measurements of a decaying magnetic eigenmode in a spherical system
PACS numbers: 47.65.+a, 47.27.-i, 52.65.Kj, 91.25.Cw

$(r=0.3 \mathrm{~m})$ [8]. They used counterrotating vortices (in the Northern and Southern Hemispheres), applied a pulsed magnetic field, and measured the decay rate.

We report the first measurement of the reduction of the conductivity of a liquid metal due to turbulence (the so-called $\beta$ effect). Liquid sodium was contained in a $0.15 \mathrm{~m}$ diameter glass sphere with baffles around the outer wall to break up toroidal flow (see Fig. 1). The magnetic Reynold's number $R_{m}=\mu_{0} \sigma_{0} L v$ was based on the tip speed of our propeller and the radius of the sphere. An effective Reynold's number $\tilde{R_{m}}$ based on the fluctuating velocity $\tilde{v}$ is lower by some factor but is difficult to measure in the liquid sodium. In addition, we measured a change in the skin depth of an oscillating magnetic field with a magnetic probe array also indicating a reduction of the conductivity.

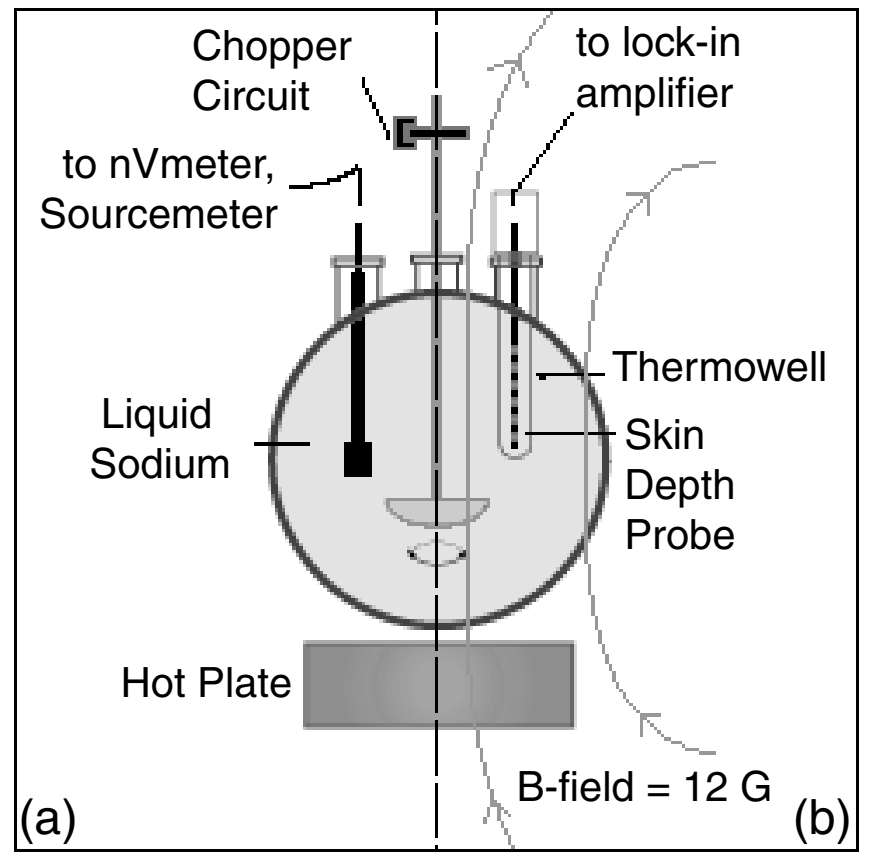

FIG. 1. Spherical sodium flow apparatus. Sodium was contained in heavy Pyrex flask $(r=0.075 \mathrm{~m})$ and flow was driven by a Teflon propeller $(r=0.035 \mathrm{~m})$. (a) Depicts one of the copper electrodes used for the dc measurement; (b) depicts the magnetic probe array used for the skin depth measurement. 
Mean-field electrodynamics predicts modifications to Ohm's law due to turbulence [10-12]. Separating the magnetic and velocity fields into mean and fluctuating parts $\left(\mathbf{B}=\mathbf{B}_{0}+\tilde{\mathbf{B}}\right.$ and $\left.\mathbf{v}=\mathbf{v}_{0}+\tilde{\mathbf{v}}\right)$ gives rise to a turbulent electric field: $\mathbf{E}_{\text {turb }}=\alpha \mathbf{B}-\beta \nabla \times \mathbf{B}$ where $\alpha$ and $\beta$ are constants calculated from the statistics of the turbulent flow. The constant $\alpha$ is related to selfexcitation and growth of the magnetic field while $\beta$ is related to enhanced magnetic diffusivity due to turbulence. We can then write

$$
\mathbf{J}=\sigma_{0}[\mathbf{E}+\mathbf{v} \times \mathbf{B}+\alpha \mathbf{B}-\beta \nabla \times \mathbf{B}],
$$

where $\sigma_{0}$ is the molecular conductivity $\left[\sigma_{0}=9.76 \times\right.$ $10^{6}(\Omega \mathrm{m})^{-1}$ for sodium at $120^{\circ} \mathrm{C}$ [13] ]. Invoking Ampere's law and ignoring the $\alpha$ term for now we find

$$
\left[1+\mu_{0} \beta \sigma_{0}\right] \mathbf{J}=\sigma_{0}[\mathbf{E}+\mathbf{v} \times \mathbf{B}] .
$$

We are then able to write an expression for the turbulent conductivity:

$$
\sigma_{\text {turb }}=\sigma_{0} /\left[1+\mu_{0} \beta \sigma_{0}\right] .
$$

The constant $\beta$ is derived from mean-field electrodynamics assuming isotropic turbulence [11]:

$$
\beta \cong\left(\tau_{\text {corr }} / 3\right)\left\langle\tilde{v}^{2}\right\rangle
$$

where $\tau_{\text {corr }}$ is the mean correlation time of a turbulent fluctuation and $\left\langle\tilde{v}^{2}\right\rangle$ is the mean square fluctuating velocity. Note that since $\tau_{\text {corr }}=L_{\text {corr }} / v$ we can write $\mu_{0} \beta \sigma_{0}$ as an effective Reynold's number $\tilde{R_{m}}$ and obtain $\sigma_{\text {turb }} \cong \sigma_{0}\left(1-\tilde{R_{m}} / 3\right)$ for small $\tilde{R_{m}}$. From analogous water experiments [14] we were able to estimate turbulent fluid properties. In our case, estimates of the rms fluctuating velocity from digital video imaging gives $\left\langle\tilde{v}^{2}\right\rangle \cong(0.2 \mathrm{~m} / \mathrm{s})^{2}$ and the correlation time $\tau_{\text {corr }}=L_{\text {corr }} / v \cong 0.1 \mathrm{~s}$ so $\beta$ should be of order $10^{-3} \mathrm{~m}^{2} / \mathrm{s}$ and we should expect turbulence to reduce the conductivity by a few percent. A more precise estimate of $\beta$ would require precise measurement of the turbulent flow field [15] and/or an accurate 3D turbulent fluid simulation.

Oxygen-free, high conductivity (OFHC) copper electrodes were machined from single cylindrical rods (no solder joints) and immersed in the flow at the midplane. Teflon insulation prevented the stalks of the electrodes (12.7 cm long) from contacting the sodium. The copper electrodes $(0.95 \mathrm{~cm}$ diameter, $1.91 \mathrm{~cm}$ long) were in good electrical contact with the liquid sodium. The system was maintained near $120^{\circ} \mathrm{C}$ in a silicone oil bath for thermal equilibrium. Temperature control of the bath was maintained to within $\pm 0.2^{\circ} \mathrm{C}$. Since the magnetic Prandtl number for sodium is small $\left(\mathrm{Pr}_{m}=8.3 \times 10^{-6}\right.$ at $\left.120^{\circ} \mathrm{C}\right)$ and the kinematic Reynold's number for our experiments was large $R=R_{m} / \operatorname{Pr}_{m} \cong 10^{6}$, we assumed uniform tempera- ture throughout the fluid. Contact potentials were avoided by constructing all hardware and connectors from copper. Corrosion and oxidation were minimized with an inert argon atmosphere. There was no evidence of electrode corrosion at the end of the experimental campaign.

The resistance of the copper electrodes was a substantial fraction of total resistance of the system (total resistance of about $270 \mu \Omega$ for both electrodes) but was subtracted from the total signal. The linear temperature resistance coefficient for copper is about $0.00393{ }^{\circ} \mathrm{C}^{-1}$ and for liquid sodium is about $0.0031{ }^{\circ} \mathrm{C}^{-1}$ [8]. So a $0.2{ }^{\circ} \mathrm{C}$ rise in temperature would raise the total measured resistance of our system by about $0.2 \mu \Omega$ (almost entirely due to the copper).

We can estimate the resistance of our sodium flow. The molecular resistivity of sodium is about $10 \mu \Omega \mathrm{cm}$ at $120^{\circ} \mathrm{C}$. The exposed electrode area was about $6.4 \mathrm{~cm}^{2}$ and the mean separation of the electrodes (i.e., the mean current path) was about $10 \mathrm{~cm}$, so about a $15 \mu \Omega$ resistance was predicted. After subtracting the known resistance of our copper electrodes, we measured about $9 \mu \Omega$. This correspondence shows that additional contact resistance at the sodium/copper interface due to poor wetting must have been low. Furthermore, when we attempted to measure the resistance of the copper electrodes alone (outside the sodium flow), the measured resistance was always higher than with the electrodes immersed in the sodium. Evidently, when the electrodes were strapped together, we were able to make contact with only a fraction of their surface area.

Direct measurement of the electrical resistance of the sodium flow was performed using a Keithley 2182 Nanovoltmeter in conjunction with a Keithley 2400 Sourcemeter. These devices were configured in such a way that the source meter would drive dc current between the electrodes (typically $\leq 0.1 \mathrm{~A}$ dc for $0.1 \mathrm{~s}$ ) and trigger the nanovoltmeter to take a measurement. The source meter then reverses the polarity of the current and the nanovoltmeter measures the reversed voltage. Finally, the nanovoltmeter reported half of the difference of the measurements (essentially the average of the two readings). We recorded this measurement over a $5 \mathrm{~min}$ period and computed the average. This technique effectively subtracts bias effects such as the inductive electric field due to the Earth's magnetic field $\mathbf{v}_{0} \times \mathbf{B}_{\text {Earth }}$ and contact potentials. Our error bars represent the standard deviation of the mean measurement.

In order to reduce systematic error during a run, we performed our scan of propeller speed at random settings between 0 and 40 revolutions per second (up to about 2400 revolutions per minute). The propeller was Teflon and the shaft and vessel were glass, so the only moving conductor in the experiment was sodium. Furthermore, the only other conductors in the experiment were the two copper electrodes. The support frame was constructed of nonmagnetic aluminum. The mechanical power delivered to the sodium in our experiment (about $40 \mathrm{~W}$ ) was small 


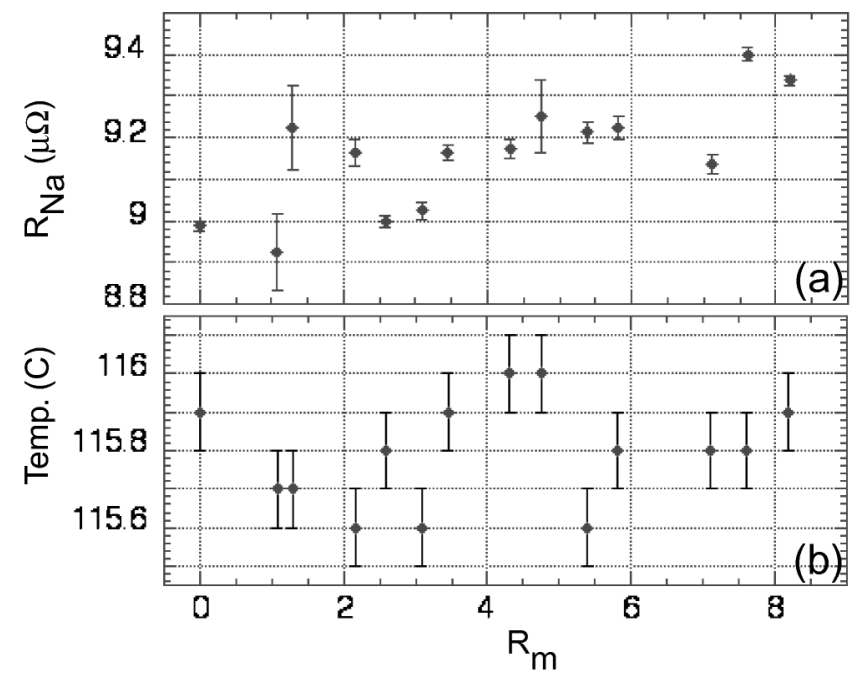

FIG. 2. (a) Measured resistance of the sodium flow $R_{\mathrm{Na}}$ as a function of $R_{m}$ with resistance of copper electrodes subtracted and zero suppressed; (b) measured temperature of the system for each resistance measurement.

compared to the heating power (about $500 \mathrm{~W}$ ). In addition, the Ohmic power delivered during our measurement was negligible (a few $\mu \mathrm{W}$ ). Nonetheless, we were careful to check that there was not a systematic increase in the measured resistance after a high velocity run due to temperature changes. Note that our operational regime was quite different from that of the Maryland group [8]. In their case, mechanical power was so high that they needed to provide cooling power to maintain temperature control.

In Fig. 2a we present the raw data from our dc experiment: measured resistance of our sodium flow as a function of $R_{m}$ with the known resistance of the copper electrodes subtracted. Again, the error bars represent the standard deviation of the mean measured resistance over a $5 \mathrm{~min}$ period and note that the zero has been suppressed. The large error bars around $R_{m}=1.5$ were due to a mechanical resonance of our apparatus at about $7 \mathrm{~Hz}$. There was another mechanical resonance at around $R_{m}=6.5(30 \mathrm{~Hz})$. Note that the increased resistance of our system was about $0.5 \mu \Omega$ and was larger than could be attributed to temperature fluctuations. In Fig. $2 \mathrm{~b}$ we present the temperature of the system measured at the time of each resistance measurement. Note that the temperature for this run was maintained constant to within $\pm 0.2^{\circ} \mathrm{C}$ (in fact, the temperature at $R_{m}=0$ and at $R_{m}=8$ were the same on this run). All other data runs (some with smaller, more resistive electrodes) showed similar trends, but the data presented here exhibited the best temperature control.

In Fig. 3a, we extracted the conductivity of the sodium flow from the resistance measurement. Measured conductivities were then normalized to the molecular value at $R_{m}=0$. Note that there was a statistically significant drop in the conductivity (about 4\%). Finally, in Fig. 3b, we computed $\beta$ from the data using Eq. (5). The $t$ value for the slope (the ratio of the estimated slope to its stan-

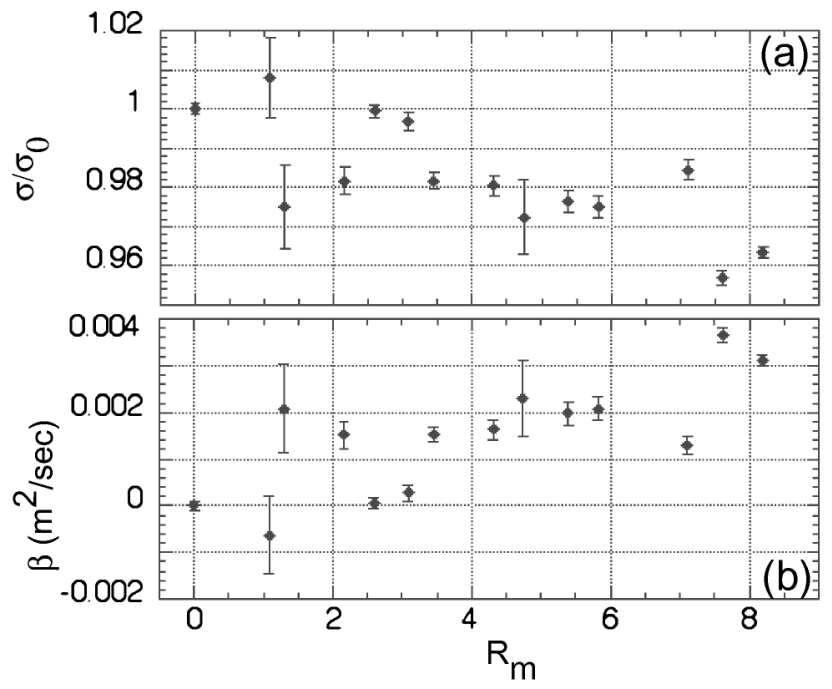

FIG. 3. (a) Normalized sodium conductivity from dc measurement as a function of $R_{m}$ (zero suppressed); (b) calculation of $\beta$ from (a) using Eq. (5).

dard error) was 7.6, which was significant to well above the $99 \%$ confidence level. Note that the measured value agrees with our order of magnitude estimate of $10^{-3} \mathrm{~m}^{2} / \mathrm{s}$.

As a secondary experiment, we measured the change in bulk conductivity through the change in the skin depth of an oscillating magnetic field with a magnetic probe array $\left(\delta \cong 1 / \sqrt{\mu_{0} \sigma \omega}\right)$. This was a useful check since it constituted a spatially averaged measurement of the conductivity. A spatially uniform but temporally oscillating magnetic field $(290 \mathrm{~Hz})$ was applied to the sodium flow with a large set of Helmholtz coils. The coils formed part of a driven resonant $\mathrm{LC}$ circuit. The oscillating magnetic field (12 G peak amplitude) was measured in the flow at
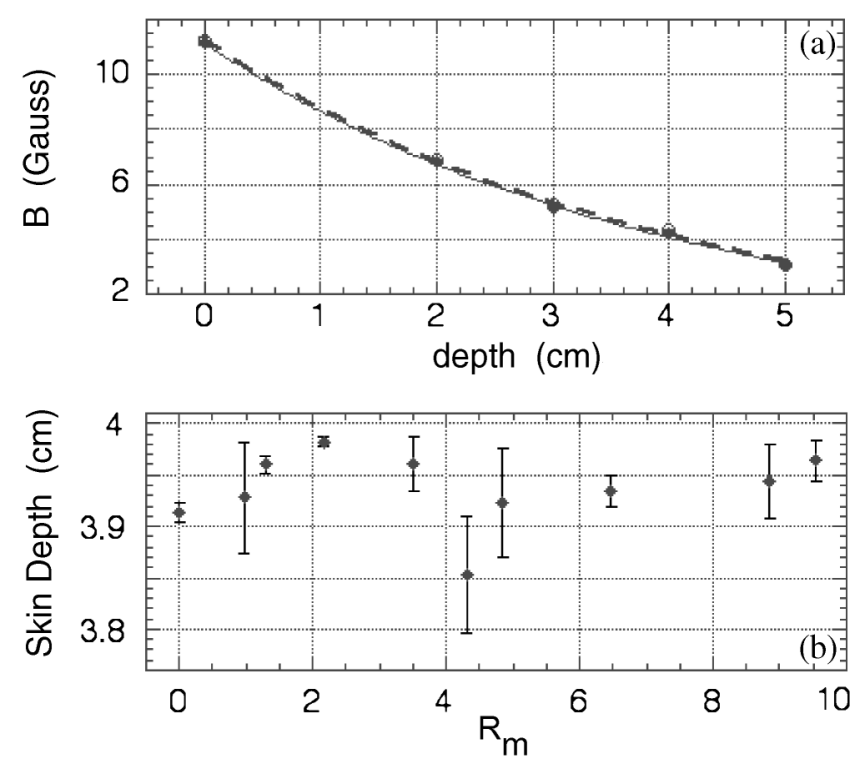

FIG. 4. Skin depth of $290 \mathrm{~Hz}$ oscillating $B$ field as a function of $R_{m}$; (a) raw data for $R_{m}=0$ (solid line) and 9 (dashed line); (b) calculated skin depth as a function of $R_{m}$. 


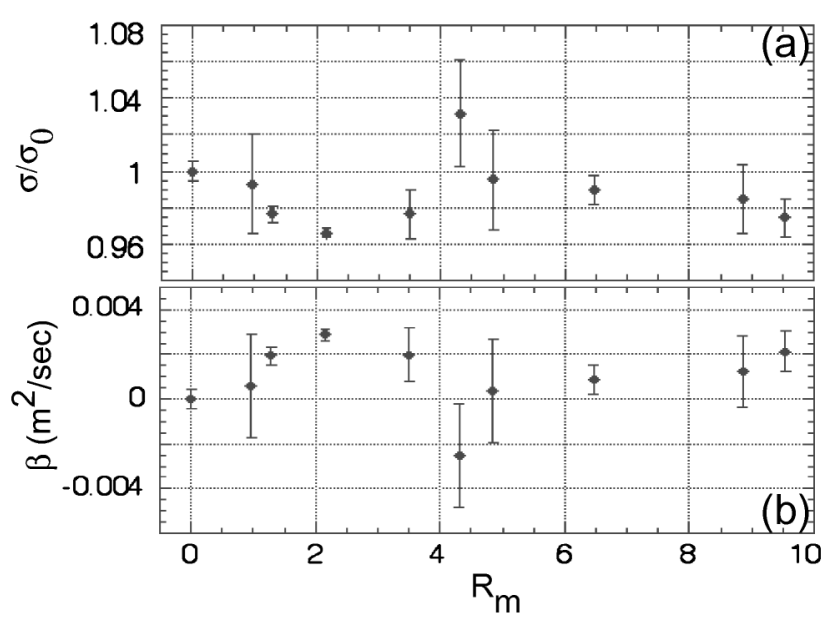

FIG. 5. Normalized sodium conductivity from skin depth measurement as a function of $R_{m}$ (zero suppressed). (b) Calculation of $\beta$ from (a) using Eq. (5).

six locations by magnetic pickup loops separated by $1 \mathrm{~cm}$ (see Fig. 1b). Signals were detected with a lock-in amplifier referenced to the oscillator driving the LC circuit and with a $3 \mathrm{~s}$ time constant. The probe array was calibrated in the uniform field region of the Helmholtz coils in the absence of sodium.

In Fig. 4a we present representative raw data: measured magnetic signal on each probe (using the lock-in amplifier) for $R_{m}=0$ and $R_{m}=9$. Fluctuations on the probe at the surface of the sodium were large so that point was discarded for the skin depth fit. In Fig. 4b, we present the calculated skin depth as a function of $R_{m}$. The error bars were computed from the standard error of the slope of the exponential and were unfortunately quite large. However, there was a trend toward longer skin depth at higher $R_{m}$.

Finally, in Fig. 5a, we present the extracted (normalized) conductivity from these data with error propagated from the data of Fig. 4. In Fig. 5b, we computed $\beta$ from the skin depth data using Eq. (5). The reduction of the mean conductivity and the increase in $\beta$ were consistent with the dc measurement (Fig. 3), but were inconclusive by themselves. The $t$ value for the slope was 1.6 corresponding to a confidence level of $94 \%$.

To summarize, we have measured the reduction of the conductivity of liquid sodium by about $4 \%$ due to turbulence at $R_{m}$ of about 8 (the so-called $\beta$ effect). Temperature control was maintained to within $0.2{ }^{\circ} \mathrm{C}$ and mechanical heating of the liquid sodium was negligible. Results roughly agree with simple predictions from mean-field electrodynamics.

This work was performed under Department of Energy (DOE) Grant No. DE-FG02-97ER54422. M. R. B. is a DOE Junior Faculty Investigator. A. B. R. acknowledges support from DESGC. The authors gratefully acknowledge the assistance of W. Luh, H. Hoffsommer, M. Dimeglio, S. Palmer, and D. Radcliff.

[1] S. I. Vainshtein et al., Astrophys. J. 404, 773 (1993).

[2] E. N. Parker, Astrophys. J. 281, 839 (1984).

[3] S. I. Vainshtein and R. Rosner, Astrophys. J. 376, 199 (1991).

[4] E. N. Parker, Astrophys. J. 408, 707 (1993).

[5] M. Steenbeck et al., Sov. Phys. Dokl. 13, 443 (1968).

[6] A. Gailitis et al., Magn. Gidrodin. 4, 3 (1987).

[7] A. Gailitis et al., Phys. Rev. Lett. 84, 4365 (2000).

[8] N. L. Peffley et al., Phys. Rev. E 61, 5287 (2000).

[9] U. Muller and R. Stieglitz, Naturwissenschaften 87, 381 (2000).

[10] M. Steenbeck, F. Krause, and K.-H. Rädler, Z. Naturforsch. 21a, 369 (1966).

[11] F. Krause and K.-H. Rädler, Mean Field Electrodynamics and Dynamo Theory (Cambridge University Press, Cambridge, U.K., 1980).

[12] H. K. Moffatt, Magnetic Field Generation in Electrically Conducting Fluids (Cambridge University Press, Cambridge, U.K., 1978).

[13] Liquid-Metals Handbook (Atomic Energy Commission, Washington, DC, 1952).

[14] A.B. Reighard, W.H. Luh, and M.R. Brown (to be published).

[15] M. D. Nornberg et al., Bull. Am. Phys. Soc. 44, 281 (1999). 